\title{
The use of inhaled corticosteroids and the risk of adrenal insufficiency
}

\author{
Francesco Lapi ${ }^{1,2,3}$, Abbas Kezouh', Samy Suissa ${ }^{1,2}$ and Pierre Ernst ${ }^{1,4}$ \\ Affiliations: \\ ${ }^{1}$ Centre for Clinical Epidemiology, Lady Davis Research Institute, Jewish General Hospital, Montreal, QC, \\ ${ }^{2}$ Dept of Epidemiology, Biostatistics and Occupational Health, McGill University, Montreal, QC, and \\ ${ }^{4}$ Dept of Medicine, McGill University, Montreal, QC, Canada. \\ ${ }^{3}$ Dept of Preclinical and Clinical Pharmacology, University of Florence, Florence, Italy.
}

\section{Correspondence:}

P. Ernst, Centre for Clinical Epidemiology, Lady Davis Research Institute, Jewish General Hospital, 3755 Cote Ste-Catherine, Montreal, QC H3T 1E2, Canada.

E-mail: pierre.ernstamcgill.ca

ABSTRACT Adrenal insufficiency is a potential complication of therapy with an inhaled corticosteroid (ICS). Although prior studies found the highest risk of adrenal insufficiency with fluticasone, a more potent ICS, these results might be explained by a channelling bias and concomitant exposure to oral corticosteroids.

We re-examined the relationship between the use of ICSs and adrenal insufficiency by using a cohort of patients treated for respiratory conditions during 1990-2005, identified in the healthcare databases from the province of Quebec, Canada, with follow-up until 2007. A nested case-control analysis was performed within this cohort. Cases of adrenal insufficiency were matched with up to 10 controls.

392 cases were identified (incidence rate 1.1 per 10000 person-years). Although the rate of adrenal insufficiency was not significantly higher among all current users of ICSs, patients receiving the highest dosages showed a greater risk (OR 1.84, 95\% CI 1.16-2.90). Consistently, an increased risk was estimated for the highest tertile of ICS dose (OR 1.90, 95\% CI 1.07-3.37) cumulated in the year before the event.

ICS at high doses appear to be a significant independent risk factor for adrenal insufficiency. Physicians prescribing ICS at such dosages should be sensitive to the signs and symptoms of adrenal insufficiency in their patients.

0

@ERSpublications

ICSs at high doses appear to be a significant independent risk factor for adrenal insufficiency http://ow.ly/l3oAC

For editorial comments see page 9 .

Received: May 232012 | Accepted after revision: Sept 282012 | First published online: Oct 112012

Conflict of interest: Disclosures can be found alongside the online version of this article at www.erj.ersjournals.com 


\section{Introduction}

Acute adrenal insufficiency is one of the most serious potential adverse effects of inhaled corticosteroids (ICSs) [1]. The frequency of its occurrence is not well known although it is said that suppression of hypothalamic-pituitary-adrenal function by chronic administration of corticosteroids, usually by the oral route, is the most common cause of adrenal insufficiency [2, 3].

This disease may be particularly likely to occur with ICSs, such as fluticasone, which have higher lipophilicity and a more prolonged half-life $[4,5]$. In a survey of UK specialists carried out by ToDD et al. [6], use of fluticasone was reported among $94 \%$ of the cases of adrenal insufficiency identified among users of ICSs. MORTIMER et al. [7] carried out a case-control analysis of cases of adrenal insufficiency within a population-based cohort in the UK, and concluded that the absolute risk for ICSs was small as compared with the use of an oral corticosteroid (OCS), although it increased with increasing ICS dose. Interestingly, the highest relative risk among ICS molecules was seen in association with a recent prescription of fluticasone. Adjustment for the concomitant use of OCSs may have been suboptimal however; that adjustment was carried out for the number of OCS prescriptions rather than cumulative dose. Moreover, fluticasone was generally prescribed by specialist physicians in the UK and may, therefore, have been channelled towards patients with more severe disease. Such channelling bias may account for the apparent association of fluticasone use with adrenal insufficiency in the UK [8]. Furthermore, subjects could be at risk of adrenal insufficiency after a prolonged exposure to ICS followed by their sudden interruption $[9,10]$. MORTIMER et al. [7] did not specifically examine the role of past use of ICSs.

Thus, because of different prescribing patterns in different countries, it may be worthwhile re-examining the relationship between the use of ICS and adrenal insufficiency in different locations to test whether an observed association might not be explained by channelling of the drug to subjects at the greater risk of experiencing the adverse events, rather than reflecting an inherent risk of the use of these medications [8, 11]. Channelling bias towards fluticasone is unlikely to occur in Canada, because fluticasone is the preferred ICS among all categories of prescribers accounting for $>75 \%$ of all ICS prescriptions [12].

In an attempt to shed further light on the association between adrenal insufficiency and use of ICSs, we carried out a nested case-control analysis in a cohort of patients identified in the health administrative databases of the province of Quebec, Canada.

\section{Patients and methods \\ Study population}

Data were obtained from the Régie de l'assurance médicale du Québec (RAMQ), which records demographic and healthcare utilisation information on all seven million residents of the province of Quebec. As of 1996, the RAMQ databases include all prescriptions for residents who belong to the provincial drug plan, a plan which has covered more than three million people since 2002 [12]. The associated MED-ECHO database [13] contains information pertaining to all hospital admissions in Quebec obtained from the data recorded by archivists on the hospitalisation discharge summary sheet. Specifically, the discharge diagnoses indicate the reasons for hospitalisation by means of primary and secondary diagnoses. The MedEcho coding rules indicate that the primary diagnosis is the most important clinical condition leading to the hospitalisation and requiring the greatest use of medical resources during the hospital stay. The secondary diagnoses may be clinically related to the primary diagnosis, comprise other concurrent diseases or are part of patients' clinical history [13].

These databases have been used previously to examine potential adverse effects of inhaled corticosteroids [14-17].

\section{Cohort definition}

A study population was defined in the RAMQ consisting of all subjects ( $n=1410211)$ who, between January 1, 1990 and December 31, 2005, were dispensed a prescription for a bronchodilator (any form of $\beta$-agonist, theophylline, ipratropium and tiotropium bromide), a cromolyn, a leukotriene antagonist, or an ICS. We formed a cohort by identifying all subjects with three or more prescriptions for these medications in any 1-year period and on at least two different dates. This was therefore a cohort of prevalent and more than occasional users of respiratory medications. Cohort entry was the date of the third prescription. Subjects with less than one complete year of RAMQ coverage before the date of their first respiratory medication prescription were excluded from the study.

We also excluded subjects for whom, in the year prior to the entry date, mention was made of adrenal insufficiency, cancer (excluding localised nonmelanoma skin cancer), diagnosis of HIV or prescription of anti-HIV medications (i.e. abacavir, atazanavir, didanosine, efavirenz, etravirine, lamivudine, lopinavir, nelfinavir, nevirapine, ritonavir, saquinavir, stavudine, tenofovir and zidovudine) and organ transplantation 
or use of anti-rejection drugs (i.e. cyclosporine, azathioprine, mycophenolate and sacrolimus) [3, 9, 10]. Furthermore, we excluded people prescribed oral hydrocortisone because this is used almost exclusively to treat existing adrenal insufficiency $[3,7,10]$, while we were interested in new cases.

All cohort members were followed from cohort entry until they left the drug plan, had an event (adrenal insufficiency), occurrence of one of the exclusion criteria, death, or until December 31, 2007 (the end of cohort follow-up), whichever came first.

\section{Case-control definition}

Cases were subjects identified using codes for hospital discharge diagnosis of adrenal insufficiency. They comprised Internation Classifiaction of Diseases (ICD)-9 codes 255.4 (corticoadrenal insufficiency) and 255.5 (other adrenal hypofunction), along with ICD-10 codes E27.1 (primary adrenocortical insufficiency), E27.2 (Addisonian crisis), E27.3 (drug-induced adrenocortical insufficiency) and E27.4 (other and unspecified adrenocortical insufficiency). These codes have been previously adopted in database studies of adrenal insufficiency $[18,19]$. Both primary and secondary diagnosis positions of the discharge summary sheets were used to identify cases.

Up to 10 controls per case were randomly selected within each risk set. Eligible controls who were alive and event-free on the calendar date of the adrenal insufficiency case (the index date) were matched to their respective cases on age ( \pm 5 years), and cohort year of entry. Matched controls were assigned the same index date as their respective case.

\section{Exposure definition}

Inhaled corticosteroid exposure included prescriptions for beclometasone, budesonide, triamcinolone, fluticasone and flunisolide, whether dispensed alone or in a combination inhaler with an inhaled $\beta$-agonist. The estimation of equivalencies were chosen on the basis of relative topical potency and what experts consider to be comparable doses according to the National Asthma Education Expert Panel report 2 (figs 3$5 \mathrm{~b}$ and $3-5 \mathrm{c}$ in that report [20]) and the Canadian asthma consensus statement (table 8 in that statement [21]). Accordingly, the equivalent doses for inhaled corticosteroids are beclometasone $100 \mu \mathrm{g}$, budesonide $80 \mu \mathrm{g}$, triamcinolone $200 \mu \mathrm{g}$, fluticasone $50 \mu \mathrm{g}$ and flunisolide $200 \mu \mathrm{g}$. All doses were converted to fluticasone equivalents and categorised according to the defined daily dose [22] of the most recent prescription in the appropriate time-window as high (fluticasone $1000 \mu \mathrm{g}$ per day), moderate (fluticasone 500-999 $\mu$ g per day) and low $(<500 \mu \mathrm{g}$ per day).

We classified ICS use during three mutually exclusive time periods, nominally the "current", "recent" and "past" time-windows. Current use was defined by a dispensing of the last prescription for an ICS within the 90-day time period immediately preceding the index date. Recent use was defined as the occurrence of the last ICS prescription dispensed at any point during the 91-180 day time-window prior to the index date, but no prescription within the 90 days prior to the event. Past exposure to ICS was defined by the dispensing of the last prescription for ICSs during the 181-365 day time period before the index date and no dispensed ICSs within 180 days before the event.

"Current use", "recent use", "past use" and "no use" of ICSs in the year before the index date were compared between cases and their matched controls, and the association between adrenal insufficiency and ICS use was estimated. Finally, exposure was further defined in terms of the most recent defined daily dose and cumulative dosage (in tertiles) among "current" and "recent" users. Such analyses of the timing in case-control analyses have allowed us previously to identify an increased risk of asthma death on cessation of ICS exposure [23], as well as the waning of the risk of pneumonia with time in patients with chronic obstructive pulmonary disease (COPD) dispensed ICSs [15].

\section{Covariates}

The covariates pertaining to risk factors for adrenal insufficiency as well as those identified as being potential confounders of the association between ICSs and adrenal insufficiency were identified from the MedEcho and RAMQ databases [12, 13].

Age and the time period in which the event occurred were adjusted for by design (matching), while sex was controlled for in the analysis. We quantified the severity of respiratory disease, independently of ICS, by the number of hospitalisations with a primary diagnosis of COPD or asthma, the number of dispensed prescriptions of respiratory medications (i.e. $\beta$-agonist, theophylline, ipratropium and tiotropium bromide, cromolyns and a leukotriene antagonist) and antibiotics, and the number of visits to a respiratory physician, all measured in the year before the index date. Furthermore, since the relationship between the outcome and exposure to ICSs is likely to be confounded by exposure to OCSs (OCSs are risk factors for adrenal insufficiency and patients prescribed high doses of ICSs are likely to have more severe respiratory disease 
and to more commonly receive OCSs), the association between ICSs and adrenal insufficiency was adjusted for the number of prescriptions or cumulative dose of OCSs in the same time-window adopted to define the exposure to ICSs. The prescriptions of oral corticosteroids were converted to prednisone (5 mg) using the following equivalent doses: cortisone $25 \mathrm{mg}$, methylprednisolone $4 \mathrm{mg}$, triamcinolone $4 \mathrm{mg}$, betamethasone $0.75 \mathrm{mg}$ and dexamethasone $0.75 \mathrm{mg}$ [24].

Other comorbidities possibly related to the risk of adrenal insufficiency were adjusted for on the basis of prescriptions for various classes of medications associated with the treatment of disorders of interest, dispensed in the past year, as we have successfully done in the past $[14,15]$. Herein, diabetes was defined on the basis of a dispensed prescription for insulin or an oral hypoglycaemic agent; cardiac disease on the basis of a prescription for cardiotropes or antihypertensives or diuretics or vasodilators; central nervous system drugs comprised benzodiazepines, major tranquillizers, anticonvulsants, antidepressants and drugs for Parkinson's disease; osteoporosis drugs included calcium, vitamin D and bisphosphonates; and rheumatic drugs included gold salts, methotrexate, azathioprine, hydroxychloroquine and chloroquine. Nonsteroidal anti-inflammatory drugs (NSAIDs) and narcotics were also considered.

\section{Data analysis}

The incidence density for the outcome of interest was computed in the cohort. Conditional logistic regression was used to compute odds ratios of adrenal insufficiency, which, for the time-matched nested case-control method used here, provides an accurate estimate of the rate ratio and the related 95\% confidence intervals [25]. Besides age, sex, calendar year of cohort entry, and duration of follow-up on which the logistic regression was conditioned, we adjusted for the confounders described above.

Two sensitivity analyses were conducted to verify the robustness of our findings.

First, all models were re-run using a more strict case definition whereby cases were restricted to only those coded as primary discharge diagnoses. Secondly, to verify the effects of underlying comorbidity and differences in age in patients with COPD or asthma, we stratified the analyses for these two conditions. According to our previous work [17], patients were classified as probable COPD if they were $\geqslant 55$ years old at cohort entry, but with no prior diagnosis (both primary or secondary positions) of asthma. All other patients were considered to have probable asthma.

Statistical analyses were conducted using SAS version 9.2 TS2M3 (SAS Institute Inc., Cary, NC, USA).

This study has received approval from the Jewish General Hospital (Montreal, QC, Canada) ethics committee.

\section{Results}

We identified a cohort of 368238 prevalent users of respiratory medications; their mean age was $49.2 \pm 27.5$ years (range $1-108)$ at cohort entry, with more females $(56.9 \%)$ than males $(43.1 \%)$. Within the cohort, 392 cases of adrenal insufficiency were identified, giving an incidence rate of 1.1 per 10000 personyears at risk.

Most of the cases $(n=342)$ were coded as secondary discharge diagnoses, and "corticoticoadrenal insufficiency" ( $n=318)$ was the most frequent code, followed by "other adrenal hypofunction" $(n=38)$, "other and unspecified adrenocortical insufficiency" ( $n=26)$, "drug-induced adrenocortical insufficiency" $(n=6)$, "primary adrenocortical insufficiency" $(n=2)$ and Addisonian crisis $(n=2)$.

Table 1 depicts demographic and clinical characteristics of cases and controls. Mean $\pm \mathrm{SD}$ age was $67.9 \pm 18.0$ years and $42.9 \%$ of cases were female. Cases had more severe respiratory disease as shown by the higher frequency of asthma and COPD hospitalisations, as well as by the higher number of prescriptions for respiratory drugs, antibiotics and cumulative dose of OCS. Other comorbidities were also more prevalent among cases.

Table 2 shows that the adjusted OR was not significantly elevated for current use (OR 1.22, 95\% CI 0.851.70 ), although the estimate appeared higher than found for recent (OR 0.78, 95\% CI 0.46-1.36) and past (OR $0.74,95 \%$ CI $0.43-1.30$ ) use. When current use was further categorised according to the dose of the most recent prescription of ICSs, the risk was significantly greater among those exposed to a high dose of ICSs (OR 1.84, 95\% CI 1.16-2.90).

Also, a significantly greater risk of adrenal insufficiency was found for the highest tertile of dosage (OR 1.90, 95\% CI 1.07-3.37) cumulated over the year before the index date (table 3). No significant associations were seen when the aforementioned analyses were conducted only among recent users of ICSs. 
TABLE 1 Characteristics of cases of adrenal insufficiency and their matched controls

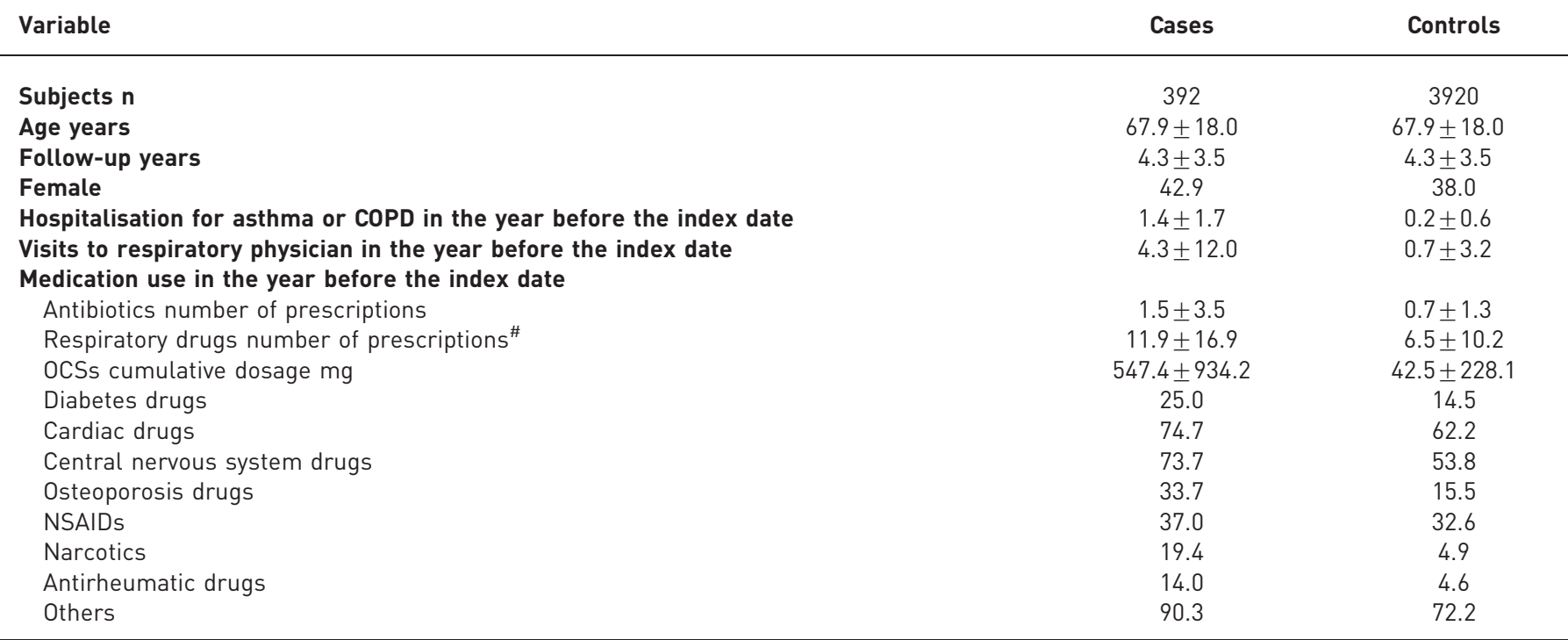

Data are presented as mean \pm SD or \%, unless otherwise stated. COPD: chronic obstructive pulmonary disease; OCS: oral corticosteroid; NSAIDs: nonsteroidal anti-inflammatory drugs. " : excluding inhaled corticosteroids.

For OCSs, the multivariable models showed that each additional prescription of OCS was associated with a $65 \%$ increased risk of adrenal insufficiency (OR 1.65, 95\% 1.52-1.82). Along this line, when the independent effect of cumulative doses of OCSs in the prior year was studied, each additional $1000 \mathrm{mg}$ of OCS was associated with a 5.2-fold time increase in risk of adrenal insufficiency (OR 5.2, 95\% CI 3.76-7.19).

The results obtained from the sensitivity analysis restricted to cases with a primary discharge diagnoses of adrenal insufficiency were consistent with the primary analysis (third tertile of cumulative dosage: OR 2.48, 95\% CI 0.40-15.33). Nevertheless, they showed wider confidence intervals including no effect because of the smaller sample size.

When we stratified the analysis by the presence of probable COPD or asthma, the risk was significantly greater among patients with COPD exposed to the current highest daily dose of ICSs (adjusted OR 2.13, 95\% CI 1.11-2.07) than those with asthma (adjusted OR 0.73, 95\% CI 0.25-2.07).

TABLE 2 Crude and adjusted odds ratios of hospitalisation for adrenal insufficiency associated with current, recent, past use and average daily dose of inhaled corticosteroids

\section{Cases Controls Crude OR $\quad$ Adjusted $^{\#}$ OR $(95 \% \mathrm{Cl})$}

\begin{tabular}{lcccc}
\hline $\begin{array}{l}\text { Subjects } \mathbf{n} \\
\text { No use in the past year }\end{array}$ & 392 & 3920 & & \\
Recency of use in the past year & 40.3 & 54.3 & Reference & Reference \\
$\quad$ Current use & & & & \\
$\quad$ Low $<500 \mu \mathrm{g}$ per day & 45.7 & 29.8 & 2.10 & $1.22(0.85-1.70)$ \\
$\quad$ Intermediate 500-999 $\mu \mathrm{g}$ per day & 3.1 & 2.6 & 1.51 & $1.72(0.83-3.57)$ \\
$\quad$ High $\geqslant 1000 \mu \mathrm{g}$ per day & 23.0 & 18.7 & 1.67 & $0.92(0.62-1.35)$ \\
Recent use & 19.6 & 8.5 & 3.24 & $1.84(1.16-2.90)$ \\
$\quad$ Low $<500 \mu \mathrm{g}$ per day & 7.7 & 7.5 & 1.37 & $0.78(0.46-1.36)$ \\
Intermediate 500-999 $\mu \mathrm{g}$ per day & 0.8 & 0.9 & 1.04 & $0.48(0.09-2.55)$ \\
$\quad$ High $\geqslant 1000 \mu \mathrm{g}$ per day & 3.6 & 4.5 & 1.07 & $0.67(0.31-1.38)$ \\
Past use & 3.3 & 2.1 & 2.77 & $0.94(0.49-2.47)$ \\
& 6.4 & 8.4 & 1.02 & $0.74(0.43-1.30)$ \\
\hline
\end{tabular}

Data are presented as \%, unless otherwise stated. ": adjusted for all factors reported in table 1;

${ }^{\top}$ : prescription within 90 days before the index date; ${ }^{+}$: prescription $91-180$ days before the index date;

$\S$ : prescription 181-365 days before the index date. 
TABLE 3 Crude and adjusted odds ratios of hospitalisation for adrenal insufficiency associated with dosages (in tertiles) of inhaled corticosteroids cumulated in the past year by the current and recent users

Cases Controls Crude OR Adjusted ${ }^{\#}$ OR $(95 \% \mathrm{Cl})$

\begin{tabular}{|c|c|c|c|c|}
\hline No use in the past year & 40.3 & 54.3 & Reference & Reference \\
\hline \multicolumn{5}{|c|}{ Recency of use in the past year as cumulative dose (tertiles) } \\
\hline \multicolumn{5}{|c|}{ Current use } \\
\hline $60000-157000 \mu \mathrm{g}$ & 15.3 & 11.1 & 1.93 & $0.96(0.60-1.52)$ \\
\hline$>157000 \mu \mathrm{g}$ & 15.3 & 6.3 & 3.57 & $1.90(1.07-3.37)$ \\
\hline \multicolumn{5}{|l|}{ Recent use $^{+}$} \\
\hline$>100000 \mu \mathrm{g}$ & 2.6 & 0.7 & 4.71 & $1.11(0.56-3.43)$ \\
\hline
\end{tabular}

Data are presented as $\%$, unless otherwise stated. ${ }^{*}$ : adjusted for all factors reported in table $1 ;{ }^{\circ}$ : prescription within 90 days before the index date; ${ }^{+}$: prescription $91-180$ days before the index date.

\section{Discussion}

In a large cohort of prevalent users of respiratory medications, we found that patients exposed to high doses of ICSs had a greater risk of developing adrenal insufficiency, whether defining dose according to the last prescription dispensed within the prior 90 days or when cumulating exposure over the prior year.

To date the evidence that ICSs are associated with adrenal insufficiency has mainly come from case reports $[6,26-28]$, and one observational study performed in the UK [7]. The risk appeared to be greater with fluticasone, but this could reflect the use of this potent agent by specialist physicians caring for more severely affected patients. Furthermore, MorTimer et al. [7] felt that the major increase in risk of adrenal insufficiency was attributable to oral rather than inhaled corticosteroids in their data [7].

In the present study, we believe that channelling bias was minimised by the fact that fluticasone is the ICS predominantly prescribed in Canada [12]. We were better able to account for the confounding effect of OCS by adjusting for cumulative dose dispensed in the prior year. Also, the Quebec databases provide information on dispensed rather than prescribed medications reducing the likelihood of exposure misclassification, which might obscure an association. We accounted for confounding by severity of respiratory disease by adjusting for the number of prescriptions for respiratory medications other than ICSs, and for exacerbation of respiratory disease as reflected by use of antibiotics, as well as hospitalisation for COPD or asthma exacerbation. However, as fluticasone is responsible for the great majority of high-dose ICS prescriptions, we were unable to examine specifically the risk of fluticasone as compared with other ICSs at equivalent doses.

We were unable to demonstrate an excess risk of adrenal insufficiency in subjects who had not refilled a prescription for an ICS in the last 3 months (recent users), which one might have expected to find as adrenal insufficiency would be expected to occur preferentially in those who have interrupted their use [10]. This may be due to our inability to precisely define interruption of the use of ICSs, as compliance with these medications is variable and therefore it is difficult to identify the actual date that the patient runs out of medication.

Interestingly, the risk of adrenal insufficiency in relation to current use of ICSs at high dose appeared higher among patients with COPD. While these results can be suggestive of an effect modification exerted by the type of respiratory disease (i.e. the different inflammatory components [29] of asthma and of COPD could modify the risk of adrenal insufficiency), this must be interpreted with caution because of the reduced sample sizes available for these sub-analyses.

This study has several limitations. First, the diagnoses of adrenal insufficiency have not been formally validated. However, the similarity in the results observed after restricting the cases to those coded as discharge diagnoses in primary position on the hospitalisation discharge summary provides some reassurance. Secondly, while we have adjusted for disease severity, it remains possible that residual confounding by severity is present. However, we do believe that the effect of ICSs is independent of the adverse effect of OCS on adrenal function as we were able to adjust for OCS use (i.e. number of prescriptions or cumulative dosage) received in the same time-windows used to define the exposure to ICSs. Thirdly, we were unable to investigate the life-time cumulative exposure to ICSs or OCS because of the 
nature of the database. Finally, although the databases contain information on drugs dispensed, we cannot guarantee that patients were actually taking the dispensed drugs. Reduced compliance could therefore attenuate the actual risk increase.

In summary, we found an increased risk of hospitalisation for adrenal insufficiency among patients who were using high doses of an ICS. The risk was smaller, however, than that observed with OCS. Although ICSs are associated with fewer adverse effects than OCS, they are not without adverse effects. They should therefore be used at their lowest effective dose and the dose decreased if asthma control has been maintained over the prior 3 months as suggested in current guidelines [30]. For patients with COPD the benefits of ICSs are more controversial [31]. The attenuated benefit as compared with asthma needs to be weighed against the potential adverse effects, including adrenal insufficiency, as fluticasone $1000 \mu \mathrm{g}$ daily, equivalent to the high doses of ICSs associated with an increased risk of adrenal insufficiency in the current study, is the ICS dose found in the combined $\beta$-agonist and ICS inhalers commonly used in COPD treatment trials.

Finally, physicians caring for patients with respiratory disease taking ICSs, especially at daily doses equivalent to fluticasone $\geqslant 1000 \mu \mathrm{g}$ per day, should be sensitive to the signs and symptoms of adrenal insufficiency in their patients.

\section{References}

Dahl R. Systemic side effects of inhaled corticosteroids in patients with asthma. Respir Med 2006; 100: 1307-1317.

2 Hopkins RL, Leinung MC. Exogenous Cushing's syndrome and glucocorticoid withdrawal. Endocrinol Metab Clin North Am 2005; 34: 371-384.

Oelkers W. Adrenal insufficiency. N Engl J Med 1996; 335: 1206-1212.

4 Hübner M, Hochhaus G, Derendorf H. Comparative pharmacology, bioavailability, pharmacokinetics, and pharmacodynamics of inhaled glucocorticosteroids. Immunol Allergy Clin North Am 2005; 25: 469-488.

5 Lipworth BJ. Systemic adverse effects of inhaled corticosteroid therapy: a systematic review and meta-analysis. Arch Intern Med 1999; 159: 941-955.

6 Todd GR, Acerini CL, Ross-Russell R, et al. Survey of adrenal crisis associated with inhaled corticosteroids in the United Kingdom. Arch Dis Child 2002; 87: 457-461.

7 Mortimer KJ, Tata LJ, Smith CJ, et al. Oral and inhaled corticosteroids and adrenal insufficiency: a case-control study. Thorax 2006; 61: 405-408.

8 Petri H, Urquhart J. Channeling bias in the interpretation of drug effects. Stat Med 1991; 10: 577-581.

9 Zwaan CM, Odink RJ, Delemarre-van de Waal HA, et al. Acute adrenal insufficiency after discontinuation of inhaled corticosteroid therapy. Lancet 1992; 340: 1289-1290.

10 Bouillon R. Acute adrenal insufficiency. Endocrinol Metab Clin North Am 2006; 35: 767-775.

11 Leufkens HG, Urquhart J, Stricker BH, et al. Channelling of controlled release formulation of ketoprofen (Oscorel) in patients with history of gastrointestinal problems. J Epidemiol Community Health 1992; 46: 428-432.

122007 IMS (IMS customer portal for Canada). www.imshealth.com/portal/site/ims?CURRENT_LOCALE=en_ca/ medi Date last accessed: February 5, 2013.

13 Santé et Services sociaux Québec. Cadre normatif du système MED-ÉCHO. http://msssa4.msss.gouv.qc.ca/fr/ document/publication.nsf/0/581d207c3ed564a7852574bf005283de?OpenDocument Date last updated: April 12, 2012. Date last accessed: February 5, 2013.

14 Ernst P, Baltzan M, Deschênes J, et al. Low-dose inhaled and nasal corticosteroid use and the risk of cataracts. Eur Respir J 2006; 27: 1168-1174.

15 Ernst P, Gonzalez AV, Brassard P, et al. Inhaled corticosteroid use in chronic obstructive pulmonary disease and the risk of hospitalization for pneumonia. Am J Respir Crit Care Med 2007; 176: 162-166.

16 Suissa S, Baltzan M, Kremer R, et al. Inhaled and nasal corticosteroid use and the risk of fracture. Am J Respir Crit Care Med 2004; 169: 83-88.

17 Suissa S, Kezouh A, Ernst P Inhaled corticosteroids, the risks of diabetes onset and progression. Am J Med 2010; 123: 1001-1006.

18 Erichsen MM, Løvås K, Fougner KJ, et al. Normal overall mortality rate in Addison's disease, but young patients are at risk of premature death. Eur J Endocrinol 2009; 160: 233-237.

19 Erichsen MM, Løvås K, Skinningsrud B, et al. Clinical, immunological, and genetic features of autoimmune primary adrenal insufficiency: observations from a Norwegian registry. J Clin Endocrinol Metab 2009; 94: $4882-4890$.

20 National Heart, Lung, and Blood Institute. Expert Panel Report 2: Guidelines for the Diagnosis and Management of Asthma. July 1997. www.nhlbi.nih.gov/about/naepp/index.htm Date last accessed: February 5, 2013.

21 Boulet LP, Becker A, Bérubé D, et al. Canadian Asthma Consensus Report, 1999. Canadian Asthma Consensus Group. CMAJ 1999; 161: S1-S61.

22 Wertheimer AI The defined daily dose system (DDD) for drug utilization review, Hosp Pharm 1986; 21: 233-234, 239-241: 258.

23 Suissa S, Ernst P, Benayoun S, et al. Low-dose inhaled corticosteroids and the prevention of death from asthma. N Engl J Med 2000; 343: 332-336.

24 Schimmer BP, Parker KL. Adrenocorticotropic hormone: adrenocortical steroids and their synthetic analogs: inhibitors of synthesis and action of adrenocortical hormones. In: Brunton LL, ed. Goodman and Gilman's Pharmacological Basis of Therapeutics. New York, McGraw-Hill, 2006.

25 Breslow NE. Statistics in epidemiology: the case-control study. J Am Stat Assoc 1996; 91: 14-28.

26 Todd GR, Acerini CL, Buck JJ, et al. Acute adrenal crisis in asthmatics treated with high-dose fluticasone propionate. Eur Respir J 2002; 19: 1207-1209.

27 White A, Woodmansee DP. Adrenal insufficiency from inhaled corticosteroids. Ann Intern Med 2004; 140: W27. 
28 Patel L, Clayton PE. Adrenal insufficiency after treatment with fluticasone. Lowest possible dose of inhaled glucocorticoids should be given. BMJ 2002; 325: 836 .

29 Bornstein SR. Predisposing factors for adrenal insufficiency. N Engl J Med 2009; 360: 2328-2339.

30 Boulet LP, Fitzgerald JM, Levy ML, et al. A guide to the translation of the Global Initiative for Asthma (GINA) strategy into improved care. Eur Respir J 2012; 39: 1220-1229.

31 Suissa S, Barnes PJ. Inhaled corticosteroids in COPD: the case against. Eur Respir J 2009; 34: 13-16. 